Pacific Journal of Mathematics

REAL CLOSURES OF FIELDS AT ORDERINGS OF HIGHER
LEVEL 


\title{
REAL CLOSURES OF FIELDS AT ORDERINGS OF HIGHER LEVEL
}

\author{
RON BROWN
}

\begin{abstract}
Natural maps are defined here which allow many questions about $\mathbf{E}$. Becker's "orderings of higher level" to be reduced to questions about the value groups of the real-valued places they induce. A simple construction is given of the set of orderings of higher level which induce a given real-valued place (this set is bijective with the set of subgroups of the value group of the place whose factor groups are cyclic of 2-power order). This construction leads to straightforward valuation-theoretic characterizations of real closed fields and of real closures of fields at orderings of higher level. The sets of isomorphism classes of real closures of a field which induce a given real-valued place, a given ordering of any level, or even a given family of orderings are each explicitly computed.
\end{abstract}

1. Introduction. Throughout this paper, $F$ will denote a field. An ordering of higher level (abbreviated: "ordering") of $F$ is a subset of $F$ which is maximal with respect to exclusion of -1 and closure under addition and multiplication (i.e., a Harrison prime) which contains $F^{2^{n}}$ for some $n$ [B]. If $T$ is such an ordering, then $T^{*}=T \backslash\{0\}$ is a subgroup of the multiplicative group $F^{*}=F \backslash\{0\}$ and $F^{*} / T^{*}$ is cyclic of order $2^{m}$ for some integer $m$ ( $m$ is called the exact level of $T$ ) [B]. Following Lam $[\mathbf{L}, \S 12]$, we will call the orderings of exact level one ordinary orderings; these are precisely the orderings appearing in the classical Artin-Schreier theory of formally real fields. Orderings of exact level greater than one will be called orderings of "higher exact level".

Orderings of higher level have received considerable attention since being introduced by Becker in 1978 [B, B1, BHR, Cr, H, KR]. Our exposition, while inevitably overlapping earlier work, is largely independent of it and is at least to some extent distinguished from it by an increased emphasis on natural constructions and mappings.

Our starting point is the fact, essentially due to Harrison and Warner [HW, Theorem 1.1] and independently rediscovered by Becker [B, p. 18], that each ordering is associated in a canonical way with a place into the field $R$ of real numbers, i.e., with a "real-valued" place.

LEMMA. Let $H$ be an ordering of $F$. There is a unique real-valued place $\tau$ on $F$ with $\tau(H) \geq 0$. 
(To deduce the lemma from [HW], first note that any subfield of the complex numbers $C$ not contained in $R$ is in fact dense in $C$ and hence clearly has elements whose $2^{n}$ th powers are not real (where $n$ is the level of our ordering $H$ ). But the residue class field of the unique place $\tau: F \rightarrow C \cup\{\infty\}$ associated with $H$ [HW, Theorem 1.1] has all its $2^{n}$ th powers in $R$ (since $\left.\tau\left(F^{2^{n}}\right) \subset \tau(H) \subset R \cup\{\infty\}\right)$. Hence $\tau$ must in fact be real-valued.)

If $\tau$ and $H$ are as in the above Lemma, we say that $H$ induces $\tau$.

Throughout this paper, $\sigma$, with associated valuation $v: F^{*} \rightarrow \Gamma$, will denote a real-valued place on $F$. The following computation of the set "X" of all orderings $H$ of $F$ inducing $\sigma$ (i.e., with $\sigma(H) \geq 0$ ) will play a fundamental role in our study. Let $\mathbf{S}$ denote the set of all subgroups $\Delta$ of $\Gamma$ such that $\Gamma / \Delta$ is cyclic of order a power of 2 .

1.1. Theorem. Let $P \in \mathbf{X}$ be an ordinary ordering. The correspondence $\psi_{P}: H \mapsto v(P \cap H)$ maps $\mathbf{X}$ bijectively onto $\mathbf{S}$.

In the statement of the above theorem we have used the convention that " $v(A)$ " will denote $v\left(F^{\circ} \cap A\right)$ for all $A \subset F$. Theorem 1.1 is proved (in a slightly stronger form) in $\S 2$, where we also show very explicitly how to construct the inverse map. Applications of it appear in [Br3], where we show that Harman's "chains of orderings" inducing $\boldsymbol{\sigma}[\mathbf{H}]$ correspond bijectively under the above map to precisely the filtrations on $\Gamma$ in the set "F" introduced below. We have very recently learned of some work of Becker, Harman and Rosenberg [BHR]. They emphasize orderings of higher level (in a generalized sense) as characters, and give independently a computation of the set $\mathbf{X}$.

A real closure of $F$ at an ordering $H$ is defined by Becker [B, p. 58] to be a maximal algebraic field extension of $F$ admitting an ordering of the same exact level as $H$ which contains $H . F$ is called real closed if it is own real closure with respect to some ordering. Real closures and real closed fields with respect to ordinary orderings are just the real closures and real closed fields of the Artin-Schreier theory [L, §3]; we will call them ordinary. The next two theorems characterize the real closures and real closed fields of higher exact level, i.e., those which are not ordinary. The first extends a theorem of Becker (cf., Remark 4.3 B) to a characterization of real closed fields of higher exact level.

1.2. THEOREM. $F$ is a real closed field of higher exact level if and only if $F$ is Henselian with respect to a real-valued place whose residue class field is an ordinary real closed field and whose value group, say $\Delta$, is such that $\Delta / n \Delta$ has exactly two elements if $n=2$ and exactly one element if $n$ is odd. 
The condition on $\Delta$ above is equivalent to saying that $\Delta$ is additively isomorphic to the direct product of a divisible group and a nontrivial pure subgroup of $I_{2}$, the additive group of 2-adic integers. (See §3.) If $\Delta$ is required to be divisible in the statement of 2.2 , we get a familiar characterization of real closed fields at ordinary orderings (e.g., see [ $\mathrm{Br} 2, \S 4])$.

In this paper, $K$ will always denote an algebraic extension of $F$.

1.3. THEOREM. $K$ is a real closure of $F$ at some ordering of higher exact level if and only if $K$ is real closed and some element of $F$ has odd value in the value group of $K$.

In the above theorem, we have called an element of an (additively written) ordered group $\Delta$ odd, if the element is not in $2 \Delta$.

The above two theorems will be proved in $\S 4$, where we will give explicit formulas for all the orderings of higher exact level of a real closure $K$ of $F$ and show that $K$ is in fact a real closure of $F$ with respect to each of these.

We next turn to the computation of "Iso", the set of all $F$-isomorphism classes of real closures of $F$ of higher exact level which induce on $F$ the real-valued place $\sigma$. To this end, let $\mathbf{P}$ denote the set of all preorders $T$ of $F$ (i.e., subsets of $F$ closed under addition and multiplication and containing $F^{\cdot 2}$ but not $\left.-1[\mathbf{L}, \S 4]\right)$ containing $\sigma^{-1}\left(R^{\cdot 2}\right)$ and with $v(T)$ of index 2 in $\Gamma$ and let $\mathbf{F}$ denote the set of all filtrations $\left(\Gamma_{i}\right)_{i \geq 0}$ of $\Gamma$ by subgroups $\Gamma_{i}$ of $\Gamma$ having $\Gamma / \Gamma_{i}$ cyclic of order $2^{i}$ for all $i \geq 0$.

\subsection{THEOREM. The correspondence}

$$
\Phi: K \mapsto\left(K^{2} \cap F,\left(v\left(K^{2^{i}} \cap F\right)\right)_{i \geq 0}\right)
$$

maps Iso bijectively onto the set of all pairs $\left(T,\left(\Gamma_{i}\right)_{i \geq 0}\right)$ in $\mathbf{P} \times \mathbf{F}$ with $v(T)=\Gamma_{1}$.

In the statement of Theorem 1.4 (and frequently below) we have denoted an element of Iso (that is, an isomorphism class) by some member of that class.

The sets $\mathbf{P}$ and $\mathbf{F}$ (and the set of $T \in \mathbf{P}$ with $v(T)$ taking on a specified value $\Gamma_{1}$ ) are discussed in Remark 2.3B and in $\S 3$ below. For example, we note there that elements of $\mathbf{F}$ correspond (almost!) bijectively to homomorphisms from $\Gamma$ to the 2-adic integers, $I_{2}$, and that elements of $\mathbf{P}$ are simply intersections of pairs of distinct ordinary orderings inducing $\sigma$. Theorem 1.4 will be proved in $\S 5$, where we will also explicitly compute 
the inverse of the bijection. We will also compute the set of real closures of $F$ inducing a given ordinary ordering of $F$, and spell out the connection between the bijections of Theorems 1.1 and 1.4. Finally, in $\S 6$, we give necessary and sufficient conditions for the existence of a real closure of $F$ inducing a given family of orderings of $F$, and we compute the set of $F$-isomorphism classes of such real closures. The main result generalizes Theorem 1.4, Becker's computation of the set of $F$-isomorphism classes of real closures of $F$ at an ordering of higher exact level [B, p. 163], and Harman's theorem on the uniqueness of chain closures $[\mathbf{H}]$.

1.5. Notation. We give here, for the convenience of the reader, a list of some notations which are used in sections other than those in which they are first introduced.

(A) $\sigma$, with associated valuation $v: F^{*} \rightarrow \Gamma$, is a real-valued place on a field $F$. $K$ is an algebraic extension of $F$.

(B) $\mathrm{X}$ is the set of orderings of $F$ inducing $\sigma$ (i.e., orderings $H$ with $\sigma(H) \geq 0), \mathbf{S}$ is the set of subgroups $\Delta$ of $\Gamma$ with $\Gamma / \Delta$ cyclic of 2-power order, and Iso is the set of $F$-isomorphism classes of real closures of $F$ at orderings of higher exact level inducing $\sigma$. For each $m \geq 1, \mathbf{X}(m)=\{H$ $\in \mathbf{X}: H$ has exact level $m\}$, and for each $m \geq 0, \mathbf{S}(m)=\{\Delta \in \mathbf{S}: \Gamma / \Delta$ has $2^{m}$ elements $\} . \mathbf{P}$ is the set of preorders $T$ on $F$ with $v(T) \in \mathbf{S}(1)$ and $T \supset \sigma^{-1}\left(R^{\cdot 2}\right)$. F is the set of filtrations $\left(\Gamma_{i}\right)_{i \geq 0}$ of $\Gamma$ with $\Gamma_{i} \in \mathbf{S}(i)$ for all $i \geq 0$.

(C) $Z, Q, R$, and $I_{2}$ denote the sets of integers, rationals, reals, and 2-adic integers, respectively. $|A|$ denotes the cardinality of the set $A$, and $A \backslash B$ the complement of the set $B$ in $A$. Finally, $S^{\cdot}$ denotes $S \backslash\{0\}$ if $S$ is an ordering, and $S^{*}$ denotes the group of multiplicative units of $S$ if $S$ is a unitary commutative ring.

2. Orderings of higher level. We now prove a slight refinement of Theorem 1.1. Let $\mathbf{X}(m)$ denote the set of orderings in $\mathbf{X}$ of exact level $m$ (for all $m \geq 1$ ) and let $\mathbf{S}(m)$ denote the set of all groups in $\mathbf{S}$, c.f., 1.5B, of index $2^{m}$ in $\Gamma$ (for all $m \geq 0$ ). For each $(P, H) \in \mathbf{X}(1) \times \mathbf{X}$ let $\psi_{P}(H)=$ $\psi(P, H)=v(P \cap H)$.

2.1. THEOREM. Let $P \in \mathbf{X}(1) . \psi_{P}$ maps $\mathbf{X}(1)$ bijectively onto $\mathbf{S}(0) \cup \mathbf{S}(1)$ and, for each $m>1, \psi_{P}$ maps $\mathbf{X}(m)$ bijectively onto $\mathbf{S}(m)$.

The proof of Theorem 2.1 will provide an explicit formula for the inverse of $\psi_{P}$ (see Remark 2.3A below). The precise dependence of the map $\psi_{P}$ upon the choice of the ordering $P$ is discussed in Remark 6.4A 
below. We might also remark here that the map $\psi$ generalizes the map $\mathbf{X}(1) \times \mathbf{X}(1) \rightarrow \operatorname{Hom}\left(\Gamma, Z^{*}\right)$ of $[\mathbf{B r}$, Proposition]. (Each homomorphism into $Z^{*}$ is determined by its kernel.) It can be generalized to map certain pairs in $\mathbf{X} \times \mathbf{X}$ (namely, the set of all $(S, T)$ with $v(S) \supset v(T)$ and $v(S) \neq v(T)$ unless $T \in \mathbf{X}(1))$ to $\mathbf{S}$. For a step in this direction see Lemma 6.3B.

We begin the proof of Theorem 2.1 by collecting some standard facts about induced real-valued places; for the convenience of the reader proofs will be sketched.

2.2. Remark. Let $H \in \mathbf{X}(m)$. Then $\sigma^{-1}\left(R^{*}\right) \cap H=\sigma^{-1}\left(R^{\cdot 2}\right)$. The inclusion $\sigma^{-1}\left(R^{*}\right) \rightarrow F^{*}$ and the valuation $v$ induce a short exact sequence

$$
1 \rightarrow R^{*} / R^{\cdot 2} \rightarrow F^{*} / H^{*} \rightarrow \Gamma / v(H) \rightarrow 0 .
$$

Finally, $v(H)$ has index $2^{m-1}$ in $\Gamma$.

Proof of 2.2 (sketch). To prove the first assertion one observes that $H \sigma^{-1}\left(R^{-2}\right)$ is a preprime and hence equals $H$ ( $H$ is maximal preprime), whence $H \supset \sigma^{-1}\left(R^{\cdot 2}\right)$. (To show that $H \sigma^{-1}\left(R^{\cdot 2}\right)$ is closed under addition note that if $a, b \in H$ and $c, d \in \sigma^{-1}\left(R^{\cdot 2}\right)$, say with $v(a) \geq v(b)$, then $a c+b d=b\left(a b^{-1} c+d\right)$ and $\sigma\left(a b^{-1} c+d\right)>0$.) To verify the exactness of (1), recall that the kernel of $v$ is $\sigma^{-1}\left(R^{*}\right)$, and the kernel of the natural map $\sigma^{-1}\left(R^{*}\right) \rightarrow F^{*} / H^{*}$ is $H^{*} \cap \sigma^{-1}\left(R^{*}\right)=\sigma^{-1}\left(R^{\cdot 2}\right)$. The last sentence of 2.2 follows from the exactness of (1).

We now prove Theorem 2.1. Note that $\psi_{P}(P)=v(P)=\Gamma \in \mathbf{S}(0)$. Let $H \in \mathbf{X}(m), H \neq P, m>0$. We show $\psi_{p}(H) \in \mathbf{S}(m)$. The natural map $P^{*} / H \cap P^{*} \rightarrow F^{*} / H^{*}$ is injective, so $P^{*} / H \cap P^{*}$ is cyclic of 2-power order. The map $P^{*} / H \cap P^{*} \rightarrow \Gamma / v(H \cap P)$ induced by $v$ is clearly surjective, so $\Gamma / v(H \cap P)$ is cyclic of 2-power order, i.e., $\psi_{P}(H) \in \mathbf{S}$. It remains to show that both the above maps are bijective, so that $\psi_{P}(H) \in$ $\mathbf{S}(m)$. The surjectivity of the first is easy $\left(P^{\cdot}\right.$ has index 2 in $F^{*}$ and $P^{*} H^{*}$ is bigger). To show that the second is injective, consider any $a \in P$ with $v(a)=v(b)$ for some $b \in H \cap P$. Then

$$
a=b\left(a b^{-1}\right) \in H \cdot\left(P \cap \sigma^{-}\left(R^{*}\right)\right)=H \cdot \sigma^{-1}\left(R^{\cdot 2}\right) \subset H,
$$

proving injectivity.

Next suppose $\Delta \in \mathbf{S}(m), m>0$. It suffices to show there exists a unique element of $\mathbf{X}(m)$ mapped by $\psi_{P}$ to $\Delta$. Set

$$
S=\left(P \cap v^{-1}(\Delta)\right) \cup-\left(P \cap v^{-1}\left(\Delta^{\prime} \backslash \Delta\right)\right)
$$


where $\Delta^{\prime}=\Delta+2^{m-1} \Gamma$. That $S$ is a subgroup of $F^{*}$ follows from the fact that $\Delta^{\prime} / \Delta$ is cyclic of order 2. (If $a, b \in P \cap v^{-1}\left(\Delta^{\prime} \backslash \Delta\right)$, then $a b \in P \cap$ $v^{-1}(\Delta)$ since $v(a b) \in\left(\Delta^{\prime} \backslash \Delta\right)+\left(\Delta^{\prime} \backslash \Delta\right) \subset \Delta$. We leave the other cases to the reader.) Clearly $-1 \notin S \supset F^{2^{m}}$ (since $v(-1) \notin v\left(\Delta^{\prime} \backslash \Delta\right)$ and $-1 \notin P$ and $\left.F^{2^{m}} \subset P \cap v^{-1}(\Delta)\right) . S$ is closed under addition: if $a, b \in S$ with $v(a) \geq v(b)$ then $a+b=b\left(1+a b^{-1}\right) \in S \cdot \sigma^{-1}\left(R^{\cdot 2}\right) \subset S$. We again have isomorphisms $P^{*} / S \cap P \rightarrow F^{*} / S$ and $P^{*} / S \cap P \rightarrow \Gamma / \Delta$ induced by the inclusion $P^{*} \rightarrow F^{*}$ and by $v$ (note that $P^{*} \neq S$ since $S \supset-\left(P \cap v^{-1}\left(\Delta^{\prime} \backslash \Delta\right)\right)$ and that $P \cap v^{-1}(\Delta)=S \cap P$ by the definition of $S)$. Thus $F^{*} / S$ is cyclic of order $2^{m}$, so $S \cup\{0\} \in \mathbf{X}(m)$ and $\psi_{P}(S \cup\{0\})=\Delta$. This proves existence. Now suppose $H \in \mathbf{X}(m)$ has $\psi_{P}(H)=\Delta$; we must show $H^{\cdot}=S$. By maximality, it suffices to show $H^{\circ} \subset S$. Since by Remark $2.2 \Gamma / v(H)$ and $\Gamma / \Delta^{\prime}$ are homomorphic images of the cyclic group $\Gamma / \Delta$ of the same order, we have $\Delta^{\prime}=v(H)$. If $a \in H \cap P$; then $a \in P \cap v^{-1}(\Delta) \subset S$. Suppose $a \in H \backslash P$. Then $v(a) \notin \Delta$ (otherwise $v(a)=v(b)$ for some $b \in H \cap P$, so $a=b\left(a b^{-1}\right.$ ) $\in P \cdot \sigma^{-1}\left(R^{\cdot 2}\right) \subset P$, a contradiction). Thus $a \in(-P) \cap v^{-1}\left(\Delta^{\prime} \backslash \Delta\right) \subset S$. The theorem is proved.

2.3. Remarks. (A) The proof of 2.1 shows that if $\Delta=\mathbf{S}(m)$ (and $\left.\Delta^{\prime}=\Delta+2^{m-1} \Gamma\right)$, then

$$
\psi_{P}^{-1}(\Delta)=\{0\} \cup\left(P \cap v^{-1}(\Delta)\right) \cup-\left(P \cap v^{-1}\left(\Delta^{\prime} \backslash \Delta\right)\right) .
$$

(B) What can be said about the sets $\mathbf{X}(1), \mathbf{P}$, and for each $\Delta \in \mathbf{S}(1)$,

$$
\mathbf{P}_{\Delta}=\{T \in \mathbf{P}: v(T)=\Delta\}
$$

which play a key role in Theorem 1.4 (and in \$5)? Theorem 2.1 gives a bijection between $\mathbf{X}(1)$ and $\operatorname{Hom}\left(\Gamma, Z^{*}\right)$ (which is bijective with $\mathbf{S}(0) \cup$ $\mathbf{S}(1)$ ). $\mathbf{P}$ is naturally bijective with the set of subsets of $\mathbf{X}(1)$ containing exactly two elements; indeed, elements of $\mathbf{P}$ are precisely the intersections of pairs of distinct elements of $\mathbf{X}(1)$. $\mathbf{P}_{\Delta}$ consists precisely of the intersections of pairs of orderings $P$ and $P^{\prime}$ in $\mathbf{X}(1)$ with $v\left(P \cap P^{\prime}\right)=\Delta$. Thus we have a natural 2:1 covering of $\mathbf{P}_{\Delta}$ by $\mathbf{X}(1)$ given by the map $P \rightarrow P \cap$ $v^{-1}(\Delta)=P \cap \psi_{P}^{-1}(\Delta)$ (for all $P \in \mathbf{X}(1)$ ).

3. Filtrations on value groups. We present some lemmas here which will be needed below. We use the notation of $\$ 1$, although the lemmas may be regarded as dealing with an arbitrary torsion free abelian group $\Gamma$.

3.1. Lemma. For any $m \geq 0$, the natural map $\mathbf{F} \rightarrow \mathbf{S}(m)$ (namely, map $\left(\Gamma_{i}\right)_{l \geq 0}$ to $\left.\Gamma_{m}\right)$ is surjective. 
Proof. It suffices to show that if $\Delta \in \mathbf{S}(m)$, then $\Delta$ contains some element of $\mathbf{S}(m+1)$. The exact sequence

$$
0 \rightarrow 2^{m} Z / 2^{m+1} Z \rightarrow Z / 2^{m+1} Z \rightarrow Z / 2^{m} Z \rightarrow 0
$$

gives rise to a surjection $\operatorname{Hom}\left(\Gamma, Z / 2^{m+1} Z\right) \rightarrow \operatorname{Hom}\left(\Gamma, Z / 2^{m} Z\right.$ ). (Apply [CE, VII 6.2, p. 136], or use [F, Theorem 27.5] to apply [M, Theorem 3.4, p. 74].) $\Delta$ is the kernel of some surjective map in $\operatorname{Hom}\left(\Gamma, Z / 2^{m} Z\right)$, which is thus induced by some surjection $g \in \operatorname{Hom}\left(\Gamma, Z / 2^{m+1} Z\right)$. Then $\Delta \supset$ ker $g \in \mathbf{S}(m+1)$.

Let $Q \Gamma$ denote some fixed divisible hull of $\Gamma$ (i.e., a minimal divisible extension of $\Gamma$ ). For the remainder of this section (and never again!) we will call a subgroup $\Delta$ of $Q \Gamma$ special if it contains $\Gamma$ but not $(1 / 2) \Gamma$, and closed if $\Delta$ is isomorphic to a direct product $D \times I$ where $D$ is divisible and $I$ is a pure subgroup of $I_{2}$ (see $1.5 \mathrm{C}$ for notation).

The next lemma describes two ways in which we can generate all the elements of $\mathbf{F}$.

3.2. LemMa. (A) Let $\Delta$ be special and closed. Then $\left(\Gamma \cap 2^{i} \Delta\right)_{i \geq 0} \in \mathbf{F}$.

(B) Let $f \in \operatorname{Hom}\left(\Gamma, I_{2}\right)$, say with $1 \in f(\Gamma)$. Then. $\left(f^{-1}\left(2^{i} I_{2}\right)\right)_{i \geq 0} \in \mathbf{F}$.

(C) Let $\left(\Gamma_{i}\right)_{i \geq 0} \in \mathbf{F}$. Then there exists $f \in \operatorname{Hom}\left(\Gamma, I_{2}\right)$ with $1 \in f(\Gamma)$ and $f^{-1}\left(2^{i} I_{2}\right)=\Gamma_{i}$ for all $i \geq 0$. Moreover, the set

$$
\Gamma^{*}:=\left\{q \gamma \in Q \Gamma: q \in Q, \gamma \in \Gamma \text { and } q f(\gamma) \in I_{2}\right\}
$$

is the unique special closed group with $\Gamma \cap 2^{i} \Gamma^{*}=\Gamma_{i}$ for all $i \geq 0$.

3.3. Note. In Lemma 3.2(C), $f$ is unique up to multiplication by a unit of $I_{2}$. Indeed the construction of 3.2(C) gives a bijection

$$
\mathbf{F} \cup\left\{(\Gamma)_{i \geq 0}\right\} \rightarrow \operatorname{Hom}\left(\Gamma, I_{2}\right) / \sim
$$

where the codomain is the set of equivalence classes of maps $f \in$ $\operatorname{Hom}\left(\Gamma, I_{2}\right)$ under the equivalence relation: $f \sim g$ if and only if $a f=b g$ for some nonzero $a, b \in I_{2}$. (Correspond the constant sequence $(\Gamma)_{i \geq 0}$ to the class of the zero map.) The point of view in this note is central to the paper $[\mathbf{B r} 3]$ and a proof of the above remark can be found there (see $[\mathbf{B r}$ 3 , §2, Claim 2]).

Proof of 3.2. (A) Write $\Delta \cong I \times D$ where $D$ is divisible and $I$ is pure in $I_{2}$. Since $\Delta$ is special, $I$ is not 2-divisible. Hence $\Delta / 2^{m} n \Delta\left(\cong I / 2^{m} I\right)$ is cyclic of order $2^{m}$ for any positive integer $m$ and odd integer $n$. Since $\Delta$ is 
special, there is a generator of $\Delta / 2^{i} \Delta$ in $\Gamma$, so that $\Gamma+2^{i} \Delta=\Delta$. Hence the natural injection $\Gamma / \Gamma \cap 2^{i} \Delta \rightarrow \Delta / 2^{i} \Delta$ is an isomorphism. Thus $\left(\Gamma \cap 2^{i} \Delta\right)_{i \geq 0} \in \mathbf{F}$, as required.

(B) Straightforward.

(C) Let $\gamma \in \Gamma \backslash \Gamma_{1}$. Then $\gamma+\Gamma_{i}$ generates $\Gamma / \Gamma_{i}$ for all $i \geq 0$. Hence there are homomorphisms $f_{i}: \Gamma \rightarrow Z / 2^{i} Z$ with $\operatorname{ker} f_{i}=\Gamma_{i}$ and $f_{i}(\gamma)=$ $1+2^{i} Z$ for all $i \geq 0$. Let $f: \Gamma \rightarrow I_{2}$ be the inverse limit of this family of maps (identify $I_{2}$ with $\lim Z / 2^{i} Z$ ), so $\operatorname{ker} f=\cap_{i \geq 0} \Gamma_{i}$ and $f(\gamma)=1$. Clearly $f^{-1}\left(2^{i} I_{2}\right)=\operatorname{ker} f_{i}=\Gamma_{i}$ for all $i \geq 0$. $f$ has a unique extension $f^{\prime}$ to a map from $Q \Gamma$ to $Q_{2}$ (the 2-adic numbers; let $f^{\prime}(\gamma / n)=f(\gamma) / n$ for $\gamma \in \Gamma$ and $0 \neq n \in Z$ ). Then $\Gamma^{*}=f^{\prime-1}\left(I_{2}\right)$. Let $f^{*}$ be the restriction of $f^{\prime}$ to $\Gamma^{*}$. The kernel of $f^{*}$ is divisible, so $\Gamma^{*}$ splits into the direct product of $\operatorname{ker}\left(f^{*}\right)$ and $f^{*}\left(\Gamma^{*}\right)$. It follows easily that $\Gamma^{*}$ is a special closed group. Moreover, $\Gamma \cap 2^{i} \Gamma^{*}=\Gamma \cap f^{*-1}\left(2^{i} I_{2}\right)=f^{-1}\left(2^{i} I_{2}\right)=\Gamma_{i}$ for all $i \geq 0$. Suppose now that $\Gamma^{\prime}$ is another special closed group with $\Gamma_{i}=\Gamma \cap 2^{i} \Gamma^{\prime}$ for all $i \geq 0$. Then $\gamma \notin 2 \Gamma^{\prime}$, so there is a homomorphism $g: \Gamma^{\prime} \rightarrow I_{2}$ with $g(\gamma)=1$ and $g^{-1}\left(2^{i} I_{2}\right)=2^{i} \Gamma^{\prime}$ for all $i \geq 0$ (argue as above with $\Gamma$ replaced by $\Gamma^{\prime}$ and $\Gamma_{i}$ by $2^{i} \Gamma^{\prime}$ ). Then $g$ restricts to $f$ on $\Gamma$ since the restriction of $g$ to $\Gamma$ induces all the maps $f_{i}$. Thus $f^{\prime}$ is the canonical extension of $g$ to a homomorphism from $Q \Gamma$ to $Q_{2}$. Hence $\Gamma^{*} \supset \Gamma^{\prime}$. That $\Gamma^{*}=\Gamma^{\prime}$ follows from the next lemma (whose proof uses only the existence part of $3.2(\mathrm{C})$ ).

The last lemma of this section contains a characterization of the value groups of real closed fields (cf., Theorem 1.2).

3.4. LEMMA. Let $\Delta$ be a special group. The following are equivalent:

(1) $\Delta$ is closed,

(2) $\Delta / n \Delta$ has 2 elements if $n=2$ and 1 element if $n$ is odd,

(3) $\Delta$ is maximal among special groups (with respect to inclusion).

Proof. " $(1) \Rightarrow(2)$ " follows from the definitions.

$(2) \Rightarrow(3)$. Suppose $\Delta^{\prime}$ is a special group containing $\Delta$. Just suppose there exists $\delta \in \Delta^{\prime} \backslash \Delta$. There exists a least positive integer $m$ with $m \delta \in \Delta$. Then $m$ is even, since otherwise $m \delta \in \Delta=m \Delta$ whence $\delta \in \Delta$. Thus $m \delta \notin 2 \Delta$ (by the choice of $m$ ). Since $\Delta^{\prime}$ is special, there exists $\gamma \in \Gamma \backslash 2 \Delta^{\prime} \subset \Delta \backslash 2 \Delta$. Since $|\Delta / 2 \Delta|=2$, we therefore have

$$
\gamma \in m \delta+2 \Delta \subset 2 \Delta^{\prime}
$$

a contradiction. 
(3) $\Rightarrow(1)$. Since $\Delta$ is special, there exists $\gamma \in \Gamma \backslash 2 \Delta$. The $Z / 2 Z$-vector space $\Delta / 2 \Delta$ has a subspace of index two missing $\gamma+2 Z$. Hence $\Delta$ has a subgroup of index 2 not containing $\Delta$. Hence there exists a filtration $\left(\Delta_{i}\right)_{i \geq 0}$ of $\Delta$ with $\Delta_{1}$ not containing $\Gamma$ and with $\Delta / \Delta_{i}$ cyclic of order $2^{i}$ for all $i \geq 0$ (apply Lemma 3.1 to $\Delta$ ). By the part of Lemma 3.2C proved above, there exists a closed subgroup $\Delta^{*}$ of $Q \Gamma$ with $\Delta^{*} \supset \Delta$ and with $\Delta \cap 2 \Delta^{*}=\Delta_{1}$. Then $\Delta^{*}$ is special, so $\Delta^{*}=\Delta$ by hypothesis. Hence $\Delta$ is closed.

4. Characterization of real closures. In this section we prove slightly strengthened versions of Theorems 1.2 and 1.3, which give valuation-theoretic characterizations of real closed fields and of real closures of a field. We continue, of course, the notation of $\S 1$.

4.1. Lemma. Let $\Gamma^{*}$ be as in Lemma 3.2(C), and let $T \in \mathbf{P}$ have $v(T)=\Gamma_{1}$. Then there exists a Henselian algebraic extension $\left(F^{*}, \sigma^{*}\right)$ of $(F, \sigma)$ such that $\sigma^{*}$ has real closed residue class field (at an ordinary ordering), $\sigma^{*}$ has value group $\Gamma^{*}$, and $F^{* 2} \cap F=T$.

Proof. Let $F_{P}$, with real-valued place $\sigma_{P}$, be a real closure of $F$ at an ordinary ordering $P$ containing $T$. Then $\sigma_{P}$ extends $\sigma$. Since $F_{P}$ is Henselian, it has a Henselian subfield $E$ with value group $\Gamma$ and (ordinary) real closed residue class field. ( $E$ is simply the Henselization of $F$ at the prime value associated with $\sigma$ [Br2, Example 1.1C and §4]. Alternately: $F_{P}$ contains a Henselization of $F$ at $v[\mathbf{R}$, p. 175] and hence an unramified extension of this with the same residue class field as $F_{P}[\mathrm{M} 1$, p. 427 "separable algebraic case"].) $E$ may be extended to a subfield $F^{*}$ of $F_{P}$ with value group $\Gamma^{*}$ by making a sequence of totally ramified extensions involving adjoining appropriate roots $a^{1 / 2^{s} m}$ in $F_{P}^{2}$, where $m$ is odd, $a \in T$, and $v(a) \in \Gamma_{s}$ for $s \geq 0$. $F^{*}$ is Pythagorean with exactly two orderings (since $\left|\Gamma^{*} / 2 \Gamma^{*}\right|=2$ ), both of which contain $T$ (since $2 \Gamma^{*} \cap \Gamma$ $=v(T)$ ). Thus $F^{* 2} \cap F=T$, as required.

4.2. THEOREM. The following three statements are equivalent:

(1) $F$ is real closed at some ordering of higher exact level;

(2) for each $m>1$, the set $F^{2^{m}} \cup-\left(F^{2^{m-1}} \backslash F^{2^{m}}\right)$ is the unique ordering of $F$ of exact level $m$ and $F$ is real closed with respect to it;

(3) $F$ is Henselian with respect to a real-valued place with an (ordinary) real closed residue class field and with a value group, call it $\Delta$, having $\Delta / n \Delta=1$ if $n$ is odd and $|\Delta / 2 \Delta|=2$. 
4.3. Remarks. (A) Groups $\Delta$ satisfying the condition in 4.2(3) were characterized in Lemma 3.4.

(B) Theorem 24 of [B, p. 58] can be easily deduced from Theorem 4.2 above and even slightly strengthened: a field $F$ which is real closed at an ordering of higher exact level is the intersection of any pair of ordinary real closed fields $K$ and $E$ lying in an algebraic closure of $F$ and containing $F$ and inducing distinct orderings on $F$. To see this, first note that $F$ has exactly two ordinary orderings (use 4.2(3) to apply either [Br] or Remark 2.3B); thus both extend to $K \cap E$. Suppose $b \in F$ has $v(b) \in 2 v(K \cap E)$. We may then assume $b$ lies in all ordinary orderings of $K \cap E$ (possibly replacing $b$ by $-b$ ), and hence in both ordinary orderings of $F$. Thus $v(b) \in 2 v(F)$. Since $v(F)$ is odd divisible, it follows that the ramification index of the extension $K \cap E$ of the Henselian field $F$ is 1 . Since the ramification degree is also clearly 1 (apply 4.2(3)), we have $K \cap E=F$ by Ostrowski's theorem [R, p. 236]. An easier application of Ostrowski's theorem also shows that $F$ has no extensions of odd degree.

(C) Here is a "relative"criterion for being real closed at an ordering of higher level: $F$ is real closed at some ordering of higher level (possibly ordinary) if and only if $F$ is Henselian with respect to a valuation with value group, call it $\Delta$, divisible by all odd integers and with a residue class field, call it $E$, which is real closed at some ordering of higher level (possibly ordinary) and which satisfies

$$
\left|E^{\cdot} / E^{\cdot 2}\right|+|\Delta / 2 \Delta| \leq 5
$$

The proof is a routine application of 4.2 and is left to the interested reader.

We now prove Theorem 4.2. First suppose $F$ is real closed at an ordering $H$ inducing $\sigma$ of exact level $m \geq 2$. Let $P \in \mathbf{X}(1)$. By Lemma 3.1 (and Theorem 2.1) there exists $\left(\Gamma_{i}\right)_{i \geq 0} \in \mathbf{F}$ with $\Gamma_{m}=v(P \cap H)$. By Lemma 4.1 we have an algebraic extension $F^{*}$ of $F$ which is Henselian at a real-valued place (say with associated valuation also denoted by $v$ ) extending $\sigma$, with value group $\Gamma^{*}$ (as in Lemma 3.2(C)), with an (ordinary) real closed residue class field, and with an ordinary ordering, $P^{*}$ say, containing $P$. By Remark 2.3A, the set

$$
\left(P^{*} \cap v^{-1}\left(2^{m} \Gamma^{*}\right)\right) \cup-\left(P^{*} \cap v^{-1}\left(2^{m-1} \Gamma^{*} \backslash 2^{m} \Gamma^{*}\right)\right) \cup\{0\}
$$

is an ordering of $F^{*}$ of exact level $m$, and its intersection with $F$ is

$$
\left(P \cap v^{-1}\left(\Gamma_{m}\right)\right) \cup-\left(P \cap v^{-1}\left(\Gamma_{m-1} \backslash \Gamma_{m}\right)\right) \cup\{0\}=H .
$$


Hence if $F$ is real closed at $H$, we must have $F=F^{*}$ (by the definition of real closure). Thus (1) implies (3). Since (2) implies (1) trivially, it remains to show that (3) implies (2). Suppose (3) holds. Let $m \geq 2$. Since $F$ admits a unique real-valued place $([\mathbf{B r 2}, \S 4]$, or $[\mathbf{P}, 7.2 .2])$, we might as well denote it by $\sigma$ and use the notation of $\S 1$. Let $P \in \mathbf{X}(1)$. Since $\mathbf{S}(m)=$ $\left\{2^{m} \Gamma\right\}$ (Lemma 3.4), the unique ordering of $F$ of exact level $m$ is

$$
\left(P \cap v^{-1}\left(2^{m} \Delta\right)\right) \cup-\left(P \cap v^{-1}\left(2^{m-1} \Gamma \backslash 2^{m} \Gamma\right)\right) \cup\{0\}
$$

(by Theorem 2.1 and Remark 2.3A), which equals $F^{2^{m}} \cup-\left(F^{2^{m-1}} \backslash F^{2^{m}}\right)$. (E.g., if $a \in P \cap v^{-1}\left(2^{m} \Gamma\right)$, then for some $b \in F ; a / b^{2^{m}} \in P \cap \sigma^{-1}\left(R^{*}\right)$ $\subset F^{2^{m}}$, so $a \in F^{2^{m}}$.) Let $H$ denote this ordering; it remains to show that $F$ is real closed at $H$. Let $(K, I)$ denote a real closure of $(F, H)$. By the proof of " $(1) \Rightarrow(3)$ " above, $K$ is Henselian at a real-valued place (say with valuation $v: K^{*} \rightarrow \Omega$ ) extending $\sigma$ which has the same residue class field as $\sigma$ does. Since $I$ and $H$ have the same exact level (cf., $\$ 1$ ), the natural map $F^{*} / H^{*} \rightarrow K^{*} / I^{*}$ is an isomorphism. Thus if $a \in F^{*}$ represents a generator of the cyclic group $F^{*} / H$; then it also represents a generator of $K^{*} / I$; and hence of its homomorphic image $\Omega / 2 \Omega$. Thus $\Omega$ contains $\Gamma$, but not $(1 / 2) \Gamma$. Hence $\Gamma=\Omega$ (Lemma 3.4). Thus $K=F$, as required [R, Ostrowski's Theorem, p. 236].

4.4. Theorem. Let $K$ be an algebraic extension of $F$, and suppose $K$ is real closed at an ordering of higher exact level. The following are equivalent:

(1) $K$ is a real closure of $F$ at some ordering of higher exact level;

(2) for each $m \geq 2$, the unique ordering of $K$ of exact level $m$ induces an ordering of $F$ of exact level $m$ and $K$ is a real closure of $F$ with respect to this ordering;

(3) some element of $F$ has odd value in the value group of $K$.

Proof. Let $w$ be the canonical valuation on $K$. Let $H$ be an ordering of $K$ of exact level $m \geq 2$. Then $H \cap F$ is an ordering of $F$, say of exact level $s$ (after all, there is a natural injection $F^{*} / F^{*} \cap H \rightarrow K^{*} / H^{*}$, so $F^{*} / F^{*} \cap H$ is cyclic of order dividing $\left.2^{m}\right) .(K, H)$ is a real closure of $(F, F \cap H)$ if and only if $s=m$. If indeed $s=m$, then $F^{*} / F^{*} \cap H \rightarrow$ $K^{*} / H^{*}$ is surjective, so $K=H F$. Hence some element of $F$ has odd value in the value group of $K$ (elements of $H$ can only take even values, i.e., values in $2 w(K))$. This shows that (1) implies (3). Conversely, suppose (3) is true. Then the natural map $w(F) / w(F \cap H) \rightarrow w(K) / w(H)$ is surjective. But $w(K) / w(H)$ has $2^{m-1}$ elements (cf., Remark 2.2), while $w(F) / w(F \cap H)$ has $2^{s-1}$ elements. Thus $2^{s-1} \geq 2^{m-1}$. Since $s$ cannot be larger than $m$, we have $s=m$. This proves (2). Since the implication “(2) $\Rightarrow(1)$ " is trivial, the Theorem is proved. 
5. Isomorphism classes of real closures. In this section we compute the set Iso of $F$-isomorphism classes of real closures of $F$ at orderings of higher exact level inducing $\sigma$, and relate this computation to that in $\$ 2$ of the orderings of $F$ inducing $\sigma$. Let us fix a subgroup $\Delta$ of $\Gamma$ of index 2 . We "relativize" some of the basic notation of $\$ 1$ by setting Iso $\mathbf{I s}_{\Delta}=\{K \in$ Iso: $\left.v\left(K^{2} \cap F\right)=\Delta\right\}, \mathbf{F}_{\Delta}=\left\{\left(\Gamma_{i}\right)_{i \geq 0} \in \mathbf{F}: \Gamma_{1}=\Delta\right\}$ and $\mathbf{P}_{\Delta}=\{T \in \mathbf{P}$ : $v(T)=\Delta\}$. The sets Iso, $\mathbf{F}$, and $\mathbf{P}$ are each the disjoint union over all $\Delta \in \mathbf{S}(1)$ of the sets $\mathbf{I s o}_{\Delta}, \mathbf{F}_{\Delta}$ and $\mathbf{P}_{\Delta}$, respectively. (E.g., each $K \in$ Iso canonically induces an element of $\mathbf{S}(1)$, namely, $v\left(K^{2} \cap F\right)$.) Thus Theorem 1.4 follows immediately from the next theorem.

5.1. THEOREM. The correspondence $K \mapsto\left(K^{2} \cap F\right.$, fil $(K)$ ) (where fil $\left.(K)=\left(v\left(K^{2^{i}} \cap F\right)\right)_{i \geq 0}\right)$ induces a bijection $\Phi_{\Delta}:$ Iso $_{\Delta} \rightarrow \mathbf{P}_{\Delta} \times \mathbf{F}_{\Delta}$.

The map $\Phi$ of Theorem 1.4 is just the union of the maps $\Phi_{\Delta}$ above. The proof of Theorem 5.1 will show that $\Phi_{\Delta}^{-1}$ is given by the construction in Lemma 4.1. For $m \geq 2$, we also set $\mathbf{S}(m)_{\Delta}=\{\Omega \in \mathbf{S}(m): \Delta \supset \Omega\}$ and $\mathbf{X}(m)_{\Delta}=\{H \in \mathbf{X}(m): v(H) \subset \Delta\}$.

Proof. Let $K$ represent an element of Iso $_{\Delta} . \sigma$ and $v$ have canonical extensions to $K$ which we also denote by $\sigma$ and $v$. $K$ is Pythagorean (Theorem $4.2(3)$ ), so $K^{2}$ is a preorder of $K$. Hence $K^{2} \cap F$ is a preorder of $F$ inducing $\boldsymbol{\sigma}$ and $\Delta$. Thus $K^{2} \cap F \in \mathbf{P}_{\Delta}$. Next, note that for all $m \geq 0$,

$$
v\left(K^{2^{m}} \cap F\right)=v(F) \cap 2^{m} v(K) .
$$

After all, if $v(a)=2^{m} v(b)$ for $a \in F$ and $b \in K$, then $a=b^{2^{m}}\left(a / b^{2^{m}}\right) \in$ $K^{2^{m}} \sigma^{-1}\left(R^{*}\right) \subset \pm K^{2^{m}}$. Hence the natural map $\Gamma / v\left(K^{2^{m}} \cap F\right) \rightarrow$ $v(K) / 2^{m} v(K)$ is not only surjective (cf., Theorem 4.4(3)), but also injective. Thus $v\left(K^{2^{m}} \cap F\right) \in \mathbf{S}(m)_{\Delta}$. Thus $\Phi_{\Delta}$ maps into $\mathbf{P}_{\Delta} \times \mathbf{F}_{\Delta}$. Now suppose $\left(T,\left(\Gamma_{i}\right)_{i \geq 0}\right) \in \mathbf{P}_{\Delta} \times \mathbf{F}_{\Delta}$. Let $\left(F^{*}, \sigma^{*}\right)$ be as in Lemma 4.1. Then $F^{*} \in \mathbf{I s 0 _ { \Delta }}$ and $\Phi_{\Delta}\left(F^{*}\right)=\left(T,\left(\Gamma_{i}\right)_{i \geq 0}\right)$ (apply 3.2(C) and (1) with $K$ replaced by $\left.F^{*}\right)$. Hence $\Phi_{\Delta}$ is surjective. Finally suppose $\Phi_{\Delta}(K)=$ $\left(T,\left(\Gamma_{i}\right)_{i \geq 0}\right)$. It remains to show that $K$ and $F^{*}$ are $F$-isomorphic. We use the notation of the proof of Lemma 4.1. We may suppose that $K \subset F_{P}$, since $K$ is contained in a real closure of $F$ at $P$ which is $F$-isomorphic to $F_{P}$. Then $K \supset E$. After all, $K$ (respectively, $E$ ) is precisely the fixed field of $F_{P}[\sqrt{-1}]$ under the group of all automorphisms $\tau$ with $\left|\sigma^{\prime}(\tau(a))\right|=$ $\left|\sigma^{\prime}(a)\right|$ for all $a \in F_{P}[\sqrt{-1}]$ which leave $K$ (respectively, $F$ ) fixed [Br2, $\S 4]$. Here, $\sigma^{\prime}$ can be either of the two complex-valued places on $F_{P}[\sqrt{-1}]$ 
which extend the unique real-valued place on $F_{P}$. (That $K \supset E$ can also be shown using the "usual" notion of Henselization [R]; an alternate proof of this sort appeared parenthetically in the proof of 4.1.) Now let $a \in P$, say with $v(a) \in \Gamma_{m}$, and let $s$ be a positive odd integer. Note that $K^{s}=K$. Let $t=2^{m}$ s. Since $v\left(K^{t} \cap F\right)=\Gamma_{m}$, there exists $c \in K \cap F_{P}^{2}$ with $c^{t} / a \in \sigma^{\prime-1}\left(R^{*}\right) \cap F_{P}^{\cdot 2} \cap F=\sigma^{-1}\left(R^{\cdot 2}\right)$. Hence $c^{t} / a=b^{t}$ for some $b \in K \cap F_{P}^{2}$ ( $K$ is Henselian). Hence $a^{1 / t}=c / b \in K$ (here, $a^{1 / t}$ denotes the unique positive $t$-th root of $a$ in $F_{P}$ ). Similarly, $a^{1 / t} \in F^{*}$. Thus $v(a) / 2^{m} s$ is in the value group of $F^{*} \cap K$. Hence $F^{*} \cap K$ is a Henselian valued field (after all, it contains $E$ ) with the same value group and residue class field as its extensions $F^{*}$ and $K$. Hence $F^{*}=F^{*} \cap K=K$ [R, Ostrowski's Theorem, p. 236].

It will be useful to formally establish a connection between the bijections $\psi_{P}: \mathbf{X} \rightarrow \mathbf{S}$ of Theorems 1.1 (and 2.1) and the bijections $\Phi_{\Delta}: \mathbf{I s o}_{\Delta} \rightarrow \mathbf{P}_{\Delta} \times \mathbf{F}_{\Delta}$ above. Let $m \geq 2$. Theorem 2.1 implies that the correspondence $(T, H) \mapsto(T, v(T \cap H))$ gives a bijection

$$
\psi_{m}: \mathbf{P}_{\Delta} \times \mathbf{X}(m)_{\Delta} \rightarrow \mathbf{P}_{\Delta} \times \mathbf{S}(m)_{\Delta} .
$$

This is because if $P \in \mathbf{X}(1)$ contains $T \in \mathbf{P}_{\Delta}$, then for any $H \in \mathbf{X}(m)_{\Delta}$, we have $\psi_{P}(H)=v(T \cap H)$ since

$$
P \cap H=P \cap v^{-1}(\Delta) \cap H=T \cap H .
$$

Next, we have a surjection $C_{m}: \mathbf{P}_{\Delta} \times \mathbf{F}_{\Delta} \rightarrow \mathbf{P}_{\Delta} \times \mathbf{S}(m)_{\Delta}$ given by $C_{m}\left(\left(T,\left(\Gamma_{i}\right)_{i \geq 0}\right)\right)=\left(T, \Gamma_{m}\right) \quad\left(\right.$ Lemma 3.1) and a map $D_{m}:$ Iso $_{\Delta} \rightarrow$ $\mathbf{P}_{\Delta} \times \mathbf{X}(m)_{\Delta}$ given by $D_{m}(K)=\left(K^{2} \cap F, H_{m}(K) \cap F\right)$ where $H_{m}(K)$ is the unique ordering of $K$ of exact level $m$ (cf., Theorem 4.4(2)). The next proposition (and the above remarks) show that the map $D_{m}$ is a surjection.

5.2. Proposition. For each $m \geq 2, C_{m} \Phi_{\Delta}=\psi_{m} D_{m}$. That is, the following diagram commutes.

$$
\begin{array}{ccc}
\mathbf{I S O}_{\Delta} & \stackrel{\Phi_{\Delta}}{\rightarrow} & \mathbf{P}_{\Delta} \times \mathbf{F}_{\Delta} \\
D_{m} \downarrow & & \downarrow C_{m} \\
\mathbf{P}_{\Delta} \times \mathbf{X}(m)_{\Delta} & \stackrel{\psi_{m}}{\rightarrow} & \mathbf{P}_{\Delta} \times \mathbf{S}(m)_{\Delta} .
\end{array}
$$

Proof. Let $K$ represent an element of $\mathbf{I s o}_{\Delta}$. Then $K^{2} \cap H_{m}(K)=K^{2^{m}}$ (cf. Theorem 4.2(2)). Hence

$$
\begin{aligned}
C_{m} \Phi_{\Delta}(K) & =\left(K^{2} \cap F, v\left(K^{2^{m}} \cap F\right)\right) \\
& =\left(K^{2} \cap F, v\left(K^{2} \cap H_{m}(K) \cap F\right)\right)=\psi_{m} D_{m}(K) .
\end{aligned}
$$


5.3. Corollary. Let $F_{P}$ be a real closure of $F$ at an ordinary ordering $P$ inducing $\sigma$.

(A) The correspondence $K \mapsto \operatorname{fil}(K)$ (cf. (5.1)) gives a bijection from the set of elements of Iso represented by subfields of $F_{P}$ to $\mathbf{F}$.

(B) Let $H \in \mathbf{X}(m), m \geq 2$. The correspondence $K \mapsto \operatorname{fil}(K)$ gives a bijection from the set of $F$-isomorphism classes of real closures of $H$ contained in $F_{P}$ to the set of $\left(\Gamma_{i}\right)_{i \geq 0} \in \mathbf{F}$ with $\Gamma_{m}=v(P \cap H)$.

Notice that (B) above (together with Lemma 3.1) implies that $H$ has at least one real closure contained in $F_{P}$ (and hence a real closure admitting an ordinary ordering extending $P$ ). The above corollary (and Theorem 5.1) are of course closely related to Becker's computation of the real closures of $F$ at an ordering of higher level [B, p. 163]. Theorem 6.1 below will generalize both the above corollary and Becker's results.

Proof. (A) The inverse map carries any $\left(\Gamma_{i}\right)_{i \geq 0} \in \mathbf{F}$ to $\Phi_{\Delta}^{-1}\left(T,\left(\Gamma_{i}\right)_{i \geq 0}\right)$ where $\Delta=\Gamma_{1}$ and $T=P \cap v^{-1}(\Delta)$ (the key fact is that if $P$ extends to $K \in$ Iso, then $K^{2} \cap F=P \cap v^{-1}(\Delta)$ where $\Delta=v\left(K^{2} \cap F\right)$ ).

(B) Suppose $K \mapsto\left(\Gamma_{i}\right)_{i \geq 0}$ under the correspondence in (A). Let $T=K^{2} \cap F$ and $\Delta=v(T)$. Then $K$ will be a real closure of $F$ at $H$ if and only if $D_{m}(K)=(T, H)$ (Theorem 4.4(2)) and hence if and only if

$$
(T, v(H \cap T))=\psi_{m} D_{m}(K)=C_{m} \Phi_{\Delta}(K)=\left(T, \Gamma_{m}\right),
$$

i.e., $v(H \cap T)=\Gamma_{m}$. But, $T=P \cap v^{-1}(\Delta)$. Thus the bijection of (A) precisely corresponds the real closures of $F$ at $H$ in $F_{P}$ to the filtrations $\left(\Gamma_{i}\right)_{i \geq 0}$ in $\mathbf{F}$ with $\Gamma_{m}=v(P \cap H)$.

6. Families of orderings. Let $\mathbf{H}$ be a nonempty subset of $\mathbf{X}$. We compute here the set of real closures of $F$ which induce all the elements of $\mathbf{H}$, i.e., real closures $K$ of $F$ such that every element of $\mathbf{H}$ is the intersection with $F$ of an ordering of $K$. We may as well suppose that we can index the elements of $\mathbf{H}$, say as $\mathbf{H}=\left(H_{i}\right)_{0 \leq i<n}$ (where $n$ is a positive integer or $\infty)$, so that if we denote the exact level of $H_{i}$ by $m(i)$, then

$$
m(0) \leq m(1)<m(2)<m(3)<\cdots
$$

and $m(0)=m(1)$ only if $m(1)=1$. (Otherwise no element of Iso will induce all the $H_{i}$, cf. $\S 4$.)

6.1. TheOREM. The set of all $K \in \mathbf{I s o}$ inducing all the elements of $\mathbf{H}$ is bijective, by the correspondence $K \mapsto \operatorname{fil}(K)$ (cf., 5.1), with the set of filtrations in $\mathbf{F}$ such that $v\left(H_{\imath} \cap H_{j}\right)$ appears as a group in the filtration whenever $0 \leq i \leq j<n$. 
When $n=1$ and $m(0) \geq 2$, the above theorem reduces to Becker's computation of the set of $F$-isomorphism classes of real closures of $F$ at $H_{0}$ [B, Theorem 13, p. 163]. Theorem 5.1 and parts (A) and (B) of Corollary 5.3 are special cases of the above theorem corresponding, respectively, to when $n=2$ and $m(0)=m(1)=1$, to when $n=m(0)=1$, and to when $n=2$ and $m(1)>m(0)=1$.

6.2. REMARK. Let $J=\{i \in Z: 0 \leq i<n\}$ and let $E$ be a nonempty subset of $J \times J$ such that $J$ and $E$ form the set of nodes and the set of edges, respectively, for a connected graph. The proof of 6.1 will show that the image of the bijection in 6.1 is precisely the set of filtrations in $\mathbf{F}$ such that $v\left(H_{i} \cap H_{j}\right)$ appears as a group in the filtration whenever $(i, j) \in E$. Thus the image of the bijection can be described as the set of filtrations in F containing $v\left(H_{i} \cap H_{0}\right)$ whenever $0 \leq i<n$ (take $E=\{(0, i): 0 \leq i<$ $n\})$ or, when $n>1$, as the set of filtrations in $\mathbf{F}$ containing $v\left(H_{i} \cap H_{i+1}\right)$ whenever $0<i+1<n$ (take $E=\{(i, i+1): 0<i+1<n\}$ ). (Theorem 6.1 itself results if we take $E=J \times J$. Strictly speaking, the sets " $E$ " above should be sets of unordered pairs of elements of $J$.)

We begin the proofs of 6.1 and 6.2 with a lemma.

6.3. Lemma. (A) Suppose $\Delta \in \mathbf{S}(m(0))$ and $v\left(H_{0}\right) \supset \Delta$. Then there exists a unique $T \in \mathbf{P}$ with $v\left(T \cap H_{0}\right)=\Delta$, namely, $T=F^{2}$. $\left(H_{0} \cap v^{-1}(\Delta)\right)$.

(B) There exists a real closure in Iso inducing both $H_{0}$ and $H_{1}$ if and only if $v\left(H_{0}\right) \supset v\left(H_{1}\right)$, in which case $v\left(H_{0} \cap H_{1}\right) \in \mathbf{S}(m(1))$.

Proof of 6.3. Let $S=F^{2}\left(H_{0} \cap v^{-1}(\Delta)\right)$. Then we claim that $-1 \notin S$. Let $P \in \mathbf{X}$ be any ordinary ordering of $F$. Set $\Upsilon=v\left(P \cap H_{0}\right)$; then $\Upsilon \in \mathbf{S}(m(0))$. By Remark 2.3A,

$$
H_{0}^{\cdot}=\left(P \cap v^{-1}(\Upsilon)\right) \cup-\left(P \cap v^{-1}\left(v\left(H_{0}\right) \backslash \Upsilon\right)\right),
$$

so that

$$
H_{0} \cap v^{-1}(\Delta)=\left(P \cap v^{-1}(\Delta \cap \Upsilon)\right) \cup-\left(P \cap v^{-1}(\Delta \backslash \Upsilon)\right) .
$$

Just suppose $-1 \in S$. Since $-1 \notin P \supset F^{2}\left(P \cap v^{-1}(\Delta \cap \Upsilon)\right)$, we have $-1 \in F^{2}\left((-P) \cap v^{-1}(\Delta \backslash \Upsilon)\right)$. Therefore $2 \Gamma \cap(\Delta \backslash \Upsilon)$ is nonempty. Hence $\Delta \neq \Upsilon$. Thus $\Delta+\Upsilon=v\left(H_{0}\right)\left(\Delta\right.$ and $\Upsilon$ are distinct subgroups of $v\left(H_{0}\right)$ of index 2). Hence $\Gamma /(\Delta+\Upsilon)$ has $2^{m(0)-1}$ elements. It follows that $\Gamma / \Delta \cap \Upsilon$ has $2^{m(0)+1}$ elements (consider the exact sequence

$$
0 \rightarrow \Gamma / \Delta \cap \Upsilon \stackrel{\varphi}{\rightarrow} \Gamma / \Delta \times \Gamma / \Upsilon \stackrel{\psi}{\rightarrow} \Gamma /(\Delta+\Upsilon) \rightarrow 0
$$


where $\varphi$ and $\psi$ are induced by the diagonal map $\Gamma \rightarrow \Gamma \times \Gamma$ and the subtraction map $\Gamma \times \Gamma \rightarrow \Gamma$, respectively). $\Gamma / \Delta \cap \Upsilon$ is not cyclic (since $\Delta / \Delta \cap \Upsilon$ and $\Upsilon / \Delta \cap \Upsilon$ are distinct subgroups with cyclic factor groups of the same order), but it does have a cyclic factor group of order $2^{m(0)}$ (namely $\Delta / \Delta \cap \Upsilon$ ). Hence $\Gamma / \Delta \cap \Upsilon$ is isomorphic to $G=\left(Z / 2^{m(0)} Z\right) \times$ $(Z / 2 Z)$. Let $\gamma \in 2 \Gamma \cap(\Delta \backslash \Upsilon)$. Since $\Delta / \Delta \cap \Upsilon$ has order 2 (consider the exact sequence $\Delta / \Delta \cap \Upsilon \rightarrow \Gamma / \Delta \cap \Upsilon \rightarrow \Gamma / \Delta), \gamma+\Delta \cap \Upsilon$ generates a subgroup of $\Gamma / \Delta \cap \Upsilon$ (namely $\Delta / \Delta \cap \Upsilon$ ) of order 2 with cyclic factor group. Hence $G$ must have an element in $2 G$ of order 2 generating a subgroup with cyclic factor group. This is easily checked to be impossible (if $2 G$ has an element of order 2 , it must be $\left(2^{m(0)-1}+2^{m(0)} Z, 0+2 Z\right)$ ). This proves our claim that $-1 \notin S$. Note that $v(S)=2 \Gamma+\Delta$, so $\Gamma / v(S)$ has two elements and $S \neq F$. But $S^{*}:=S \backslash\{0\}$ is a subgroup of $F^{*}$ containing $F^{\cdot 2} \sigma^{-1}\left(R^{\cdot 2}\right)$, so $S$ is a preorder [L, Proposition 11.5] (or argue as in the proof of [BK, Lemma 7, p. 169]). From the exact sequence

$$
1 \rightarrow \sigma^{-1}\left(R^{*}\right) / S \cap \sigma^{-1}\left(R^{*}\right) \rightarrow F^{*} / S^{*} \rightarrow \Gamma / v(S) \rightarrow 1
$$

we deduce that $\left|F^{*} / S^{*}\right|=4$. Hence $S \in \mathbf{P}$. Clearly $v\left(S \cap H_{0}\right) \supset \Delta$, so $v\left(S \cap H_{0}\right)=\Delta$ (they have the same index in $\Gamma$, cf., Theorem 2.1 and formula (2) in §5). If $v\left(T \cap H_{0}\right)=\Delta$ for some $T \in \mathbf{P}$, then $T \supset$ $F^{2}\left(H_{0} \cap T\right) \supset F^{2}\left(H_{0} \cap v^{-1}(\Delta)\right)=S$ (cf., Remark 2.3A and formula (2) of §5). Hence $T=S$; (A) is proved. Next suppose $K \in$ Iso induces $H_{0}$ and $H_{1}$; then $v\left(H_{0}\right)=v\left(K^{2^{m(0)-1}} \cap F\right) \supseteq v\left(K^{2^{m(1)-1}} \cap F\right)=v\left(H_{1}\right)$ (Theorem 4.2) and $v\left(H_{0} \cap H_{1}\right)=v\left(K^{2^{m(1)}} \cap F\right) \in \mathbf{S}(m(1))$. Conversely, suppose that $v\left(H_{0}\right) \supset v\left(H_{1}\right)$. If $H_{1}$ is an ordinary ordering, then so is $H_{0}$, and so clearly some $K \in$ Iso induces both (Theorem 5.1). Otherwise, $m(1)>m(0)$, so by (A) above there exists a $T \in \mathbf{P}$ with $v\left(T \cap H_{0}\right)=$ $2^{m(0)} \Gamma+v\left(H_{1}\right)$. There exists $\left(\Gamma_{i}\right)_{l \geq 0} \in \mathbf{F}$ with $v\left(T \cap H_{1}\right)=\Gamma_{m(1)}$ (Theorem 2.1 and Lemma 3.1). Clearly $\Phi^{-1}\left(T,\left(\Gamma_{\imath}\right)_{i \geq 0}\right)$ induces both $H_{0}$ and $H_{1}$ (Proposition 5.2).

6.4. Remarks. (A) How do the bijections $\psi_{P}: \mathbf{X} \rightarrow \mathbf{S}$ of Theorem 1.1 depend on the choice of $P$ ? First, if $P, P^{\prime} \in \mathbf{X}(1)$, then $\psi_{P}$ and $\psi_{P^{\prime}}$ agree on the set of orderings $J \in \mathbf{X}$ with $v(J) \subset \Delta$ where $\Delta=v\left(P \cap P^{\prime}\right)$ (cf., the discussion of $\psi_{m}$ preceding Proposition 5.2). Secondly, if $P \in \mathbf{X}(1)$ and $J \in \mathbf{X}$, there exists a unique $P^{\prime} \in \mathbf{X}(1)$ such that $\psi_{P}(J)=\psi_{P^{\prime}}(J)$ (apply 6.3A with $H_{0}=J$ and $\Delta=\psi_{P}(J)$; then $P$ and $P^{\prime}$ are the unique ordinary orderings containing the unique $T \in \mathbf{P}$ with $v(T \cap J)=\Delta)$. 
(B) Suppose $0 \leq i \leq j<n$. Suppose $v\left(H_{i} \cap H_{j}\right)$ is one of the groups in a filtration $\left(\Gamma_{m}\right)_{m \geq 0}$ in $\mathbf{F}$ (cf., Theorem 6.1 and Remark 6.2). By the basic properties of cyclic groups, $v\left(H_{i}\right)$ and $v\left(H_{j}\right)$ are comparable and hence, by Lemma 6.3B, if $i<j$ then $v\left(H_{i} \cap H_{j}\right)=\Gamma_{m(j)}$. If $i=j$, then $v\left(H_{i} \cap H_{j}\right)=\Gamma_{m(j)-1}($ Remark 2.2).

We now prove Theorem 6.1 and Remark 6.2. Suppose $K$ and $K^{\prime}$ in Iso both induce all the orderings in $\mathbf{H}$, and that $\Phi(K)=\left(T,\left(\Gamma_{i}\right)_{0 \leq i}\right)$ and $\Phi\left(K^{\prime}\right)=\left(T^{\prime},\left(\Gamma_{i}\right)_{i \geq 0}\right)$. Then $v\left(T^{\prime} \cap H_{0}\right)=\Gamma_{m(0)}=v\left(T \cap H_{0}\right)$ (apply 5.2 if $m(0)>1)$. Thus $T^{\prime}=F^{2} \cdot\left(H_{0} \cap v^{-1}\left(\Gamma_{m(0)}\right)\right)=T($ Lemma 6.3A). Hence $K$ and $K^{\prime}$ are $F$-isomorphic (Theorem 1.4). This shows the map in 6.1 is injective; it remains to compute its image. By Theorem 4.2(2), $v\left(H_{i} \cap H_{j}\right)$ is $\Gamma_{m(j)}$ (if $0 \leq i<j<n$ ) or $\Gamma_{m(j)-1}$ (if $0 \leq i=j<n$ ). This shows that if $\left(\Gamma_{m}\right)_{m \geq 0}$ is any filtration in the image of the map in 6.1 then $v\left(H_{i} \cap H_{j}\right)$ equals an element of $\left(\Gamma_{m}\right)_{m \geq 0}$ whenever $0 \leq i \leq j<n$, i.e., whenever $(i, j)$ is an edge in the (connected) full graph on $\{i: 0 \leq i<n\}$. Conversely (cf., Remark 6.2) suppose $\left(\Gamma_{m}\right)_{m \geq 0}$ is a filtration in $\mathbf{F}$ such that $v\left(H_{i} \cap H_{j}\right)$ equals an element of $\left(\Gamma_{m}\right)_{m \geq 0}$ for all $(i, j)$ in the set of edges $E \neq \varnothing$ of some connected graph with nodes $J=\{i: 0 \leq i<n\}$. Then for any node $i \in J$ there exists $j \in J$ with $(i, j) \in E$ and hence with $v\left(H_{i} \cap H_{j}\right)$ equal to an element of $\left(\Gamma_{m}\right)_{m \geq 0}$. Hence $v\left(H_{i}\right) \supset \Gamma_{m(i)}$ (by the properties of cyclic groups and the fact that $v\left(H_{i}\right)$ has index $2^{m(i)-1}$ in $\Gamma$ ). Thus there exists $T_{i} \in \mathbf{P}$ with $v\left(T_{i} \cap H_{i}\right)=\Gamma_{m(i)}$ (Lemma 6.3A). If $(i, j) \in E$ with $i<j$ then by Lemma 6.3,

$$
T_{i}=F^{2}\left(H_{i} \cap v^{-1}\left(\Gamma_{m(i)}\right)\right) \supseteq F^{2}\left(H_{j} \cap v^{-1}\left(\Gamma_{m(j)}\right)\right)=T_{j},
$$

(To obtain the middle inclusion, note that by 6.3B, $v\left(H_{i} \cap H_{j}\right) \in \mathbf{S}(m(j))$, so $v\left(H_{i} \cap H_{j}\right)=\Gamma_{m(j)}$. Then if $a \in H_{j} \cap v^{-1}\left(\Gamma_{m(j)}\right)$, we have $v(a)=v(b)$ for some $b \in H_{i} \cap H_{j}$, whence $a=b(a / b) \in H_{i} \cdot \sigma^{-1}\left(R^{\cdot 2}\right) \subset H_{i}$.) Thus $T_{i}=T_{j}$. Since the graph (with edges $E$ ) is connected we conclude that there exists $S \in \mathbf{P}$ with $S=T_{i}$ whenever $0 \leq i<n$. Since $v\left(S \cap H_{i}\right)=$ $\Gamma_{m(i)}$ whenever $0 \leq i<n$, we conclude that $\Phi^{-1}\left(S,\left(\Gamma_{m}\right)_{m \geq 0}\right)$ induces all the elements of $\mathbf{H}$ (Proposition 5.2).

6.5. COROLlary. The following are equivalent:

(1) Some element of Iso induces all the orderings in $\mathbf{H}$;

(2) $v\left(H_{i} \cap H_{0}\right) \supset v\left(H_{i+1}\right)$ whenever $0<i+1<n$;

(3) $v\left(H_{i-1} \cap H_{i}\right) \supset v\left(H_{i} \cap H_{i+1}\right)$ whenever $1<i+1<n$, and $v\left(H_{i}\right) \supset v\left(H_{i+1}\right)$ whenever $0<i+1<n$. 
Proof. Suppose (1) is true. Then by Theorem 6.1, $v\left(H_{i} \cap H_{0}\right)$ and $v\left(H_{i+1}\right)$ both lie in some filtration in $\mathbf{F}$; that $v\left(H_{i} \cap H_{0}\right)$ contains $v\left(H_{i+1}\right)$ follows then from the fact that its index in $\Gamma$ is not larger than that of $v\left(H_{i+1}\right)$. That (1) implies (3) is proved similarly. Conversely suppose (2) holds. Then

$$
v\left(H_{0}\right) \supset v\left(H_{i} \cap H_{0}\right) \supset v\left(H_{i+1}\right) \supset v\left(H_{i+1} \cap H_{0}\right) \in \mathbf{S}
$$

whenever $0<i+1<n$ (use Lemma 6.3B for the last assertion). Then some element of $\mathbf{F}$ contains all the groups $v\left(H_{i} \cap H_{0}\right), 0 \leq i<n$ (apply 3.1 if $n<\infty$ ). Thus $\mathbf{H}$ is in the image of the bijection of 6.1 (see Remark 6.2). Similarly, (3) implies (1) (the hypothesis $v\left(H_{i}\right) \supset v\left(H_{i+1}\right)$ is included to guarantee that $\left.v\left(H_{i} \cap H_{i+1}\right) \in \mathbf{S}\right)$.

6.6. Corollary. Any real closure of $F$ is determined up to isomorphism by any infinite set of orderings it induces on $F$. Thus if $\mathbf{H}$ is infinite, at most one real closure ( up to F-isomorphism) induces $\mathbf{H}$.

Proof. If $K \in$ Iso induces $\left(H_{i}\right)_{0 \leq i<\infty}$, then $K=\Phi_{\Delta}^{-1}\left(T,\left(\Gamma_{i}\right)_{i \geq 0}\right)$ where $\Delta=2 \Gamma+v\left(H_{3}\right)$, where $T=F^{2}\left(H_{0} \cap v^{-1}(\Delta)\right)$ (Lemma 6.3(A)), and where $\left(\Gamma_{i}\right)_{i \geq 0}$ is uniquely determined by the conditions that $v\left(H_{i}\right)=$ $\Gamma_{m(i)-1}$ for all $i \geq 0$.

6.7. Corollary. Suppose $1<n<\infty$ and $\mathbf{H}$ is induced by some element of Iso. The set of elements of Iso inducing $\mathbf{H}$ is bijective (by the usual map $K \mapsto \operatorname{fil}(K))$ with the set of all $\left(\Gamma_{i}\right)_{i \geq 0} \in \mathbf{F}$ with $\Gamma_{m(n-1)}=$ $v\left(H_{0} \cap H_{m(n-1)}\right)$. Moreover all the elements of Iso which induce $\mathbf{H}$ induce on $F$ precisely the same set of orderings of degree i for all $i \leq m(n-1)$.

Proof. The first assertion follows from Theorem 6.1 (and Lemma 6.3(B)). The second follows from Lemma 6.3(A) (for orderings of exact level 1) and Proposition 5.2 and Theorem 2.1 (for orderings of higher exact level).

\section{REFERENCES}

[B] E. Becker, Hereditarily-Pythagorean Fields and Orderings of Higher Level, IMPA Lecture Notes, No. 29, Rio de Janeiro, 1978.

[B1] L Local global theorems for diagonal forms of higher degree, J. Reine Angew. Math., 318 (1980), 36-50.

[BHR] E. Becker, J. Harman and A. Rosenberg, Signatures of fields and extension theory, J. reine angew. Math., 330 (1982), 53-75.

[BK] E. Becker and E. Köpping, Reduzierte quadratische Formen und Semiordnungen reeller Körper, Abh. Math. Sem. Univ. Hamburg, 46 (1977), 143-177. 
[BR] R. Brown, Real places and ordered fields, Rocky Mountain J. Math., 1 (1971), 633-636.

[BR1] _ An approximation theorem for extended prime spots, Canad. J. Math., 24 (1972), 167-184.

[BR2] Extended prime spots and quadratic forms, Pacific J. Math., 51 (1974), 379-395.

[BR3] The behavior of chains of orderings under field extensions and places, Pacific J. Math., 127 (1987), 281-297.

[CE] H. Cartan and S. Eilenberg, Homological Algebra, Princeton University Press, Princeton, 1956.

[CR] T. Craven, Orderings of higher level and semilocal rings, Math. Z., 176 (1981), 577-581.

[F] L. Fuchs, Infinite Abelian Groups, v. 1, Academic Press, New York, 1970.

[H] J. Harman, Chains of higher level orderings, Ph. D. Dissertation, University of California at Berkeley, 1980.

[HW] D. Harrison and H. Warner, Infinite primes of fields and completions, Pacific J. Math., 45 (1973), 201-206.

[KR] J. Kleinstein and A. Rosenberg, Witt rings of higher level, Bull. Inst. Math. Acad. Sinica, 8 (1980), 353-363.

[L] T. Y. Lam, The theory of ordered fields, Proceedings of the Algebra and Ring Theory Conference (ed. B. McDonald), University of Oklahoma 1979, Marcel Decker, 1980.

[M] S. MacLane, Homology, Springer-Verlag, New York, 1963.

[M1] _ Subfields and automorphism groups of p-adic fields, Annals of Math., 40 (1939), 423-442.

[P] A. Prestel, Lectures on Formally Real Fields, Monografias de Matemática No. 22, Instituto de Matemática Pura e Aplicada, Rio de Janeiro, 1975.

[R] P. Ribenboim, Théorie des Valuations, Les Presses de l'Université de Montréal, Montreal, 1964.

Received May 19, 1981 and in revised form August 16, 1985. Partially supported by National Science Foundation Grant MCS 8002268.

UNIVERSITY OF HAWAII

HoNOLULU, HI 96822 



\section{PACIFIC JOURNAL OF MATHEMATICS EDITORS}

\author{
V. S. VARADARAJAN \\ (Managing Editor) \\ University of California \\ Los Angeles, CA 90024 \\ HeRbert Clemens \\ University of Utah \\ Salt Lake City, UT 84112 \\ R. FINN \\ Stanford University \\ Stanford, CA 94305
}

\author{
HERMANN FLASCHKA \\ University of Arizona \\ Tucson, AZ 85721 \\ RAMESH A. GANGOLLI \\ University of Washington \\ Seattle, WA 98195 \\ VAUGHAN F. R. JONES \\ University of California \\ Berkeley, CA 94720 \\ ROBION KIRBY \\ University of California \\ Berkeley, CA 94720
}

\author{
C. C. MOORE \\ University of California \\ Berkeley, CA 94720 \\ H. SAMELSON \\ Stanford University \\ Stanford, CA 94305 \\ HAROLD STARK \\ University of California, San Diego \\ La Jolla, CA 92093
}

\section{ASSOCIATE EDITORS}
R. Arens
E. F. BECKENBACH
B. H. NEUMANN
F. WOLF
K. YosHIDA (1906-1982)

\section{SUPPORTING INSTITUTIONS}

UNIVERSITY OF ARIZONA
UNIVERSITY OF BRITISH COLUMBIA
CALIFORNIA INSTITUTE OF TECHN
UNIVERSITY OF CALIFORNIA
MONTANA STATE UNIVERSITY
UNIVERSITY OF NEVADA, RENO
NEW MEXICO STATE UNIVERSITY
OREGON STATE UNIVERSITY

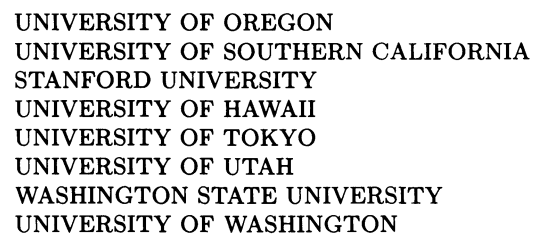

The Supporting Institutions listed above contribute to the cost of publication of this Journal, but they are not owners or publishers and have no responsibility for its content or policies.

Mathematical papers intended for publication in the Pacific Journal of Mathematics should be in typed form or offset-reproduced (not dittoed), double spaced with large margins. Please do not use built up fractions in the text of the manuscript. However, you may use them in the displayed equations. Underline Greek letters in red, German in green, and script in blue. The first paragraph must be capable of being used separately as a synopsis of the entire paper. In particular it should contain no bibliographic references. Please propose a heading for the odd numbered pages of less than 35 characters. Manuscripts, in triplicate, may be sent to any one of the editors. Please classify according to the scheme of Math. Reviews, Index to Vol. 39. Supply name and address of author to whom proofs should be sent. All other communications should be addressed to the managing editor, or Elaine Barth, University of California, Los Angeles, California 90024.

There are page-charges associated with articles appearing in the Pacific Journal of Mathematics. These charges are expected to be paid by the author's University, Government Agency or Company. If the author or authors do not have access to such Institutional support these charges are waived. Single authors will receive 50 free reprints; joint authors will receive a total of 100 free reprints. Additional copies may be obtained at cost in multiples of 50 .

The Pacific Journal of Mathematics is issued monthly as of January 1966. Regular subscription rate: $\$ 190.00$ a year (5 Vols., 10 issues). Special rate: $\$ 95.00$ a year to individual members of supporting institutions.

Subscriptions, orders for numbers issued in the last three calendar years, and changes of address should be sent to Pacific Journal of Mathematics, P.O. Box 969, Carmel Valley, CA 93924, U.S.A. Old back numbers obtainable from Kraus Periodicals Co., Route 100, Millwood, NY 10546.

The Pacific Journal of Mathematics at P.O. Box 969, Carmel Valley, CA 93924 (ISSN 0030-8730) publishes 5 volumes per year. Application to mail at Second-class postage rates is pending at Carmel Valley, California, and additional mailing offices. Postmaster: send address changes to Pacific Journal of Mathematics, P.O. Box 969, Carmel Valley, CA 93924.

PUBLISHED BY PACIFIC JOURNAL OF MATHEMATICS, A NON-PROFIT CORPORATION Copyright (C) 1987 by Pacific Journal of Mathematics 


\section{Pacific Journal of Mathematics \\ Vol. 127, No. $2 \quad$ February, 1987}

Richard Martin Aron and Robert Henry Lohman, A geometric function determined by extreme points of the unit ball of a normed space .... . . 209

Kari Astala and M. S. Ramanujan, $(s)$-nuclear sets and operators $\ldots \ldots \ldots 233$ Earl Robert Berkson, Thomas Alastair Gillespie and Paul Scott Muhly, Analyticity and spectral decompositions of $L^{p}$ for compact abelian

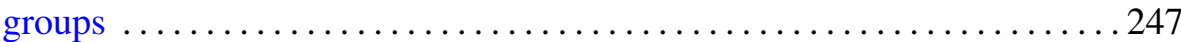

Ronald P. Brown, Real closures of fields at orderings of higher level . . . . . 261

Ronald P. Brown, The behavior of chains of orderings under field

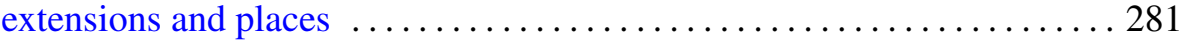

Rodney Graham Downey, Subsets of hypersimple sets .............. 299

Manfred Droste and Saharon Shelah, On the universality of systems of

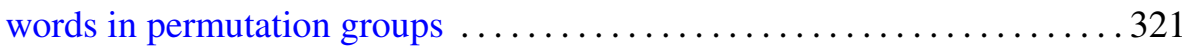

Hidenori Fujiwara, Représentations monomiales des groupes de Lie

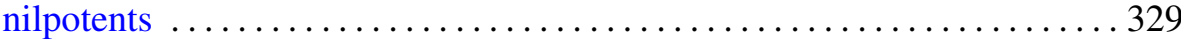

Rupert Lasser, Convolution semigroups on hypergroups $\ldots \ldots \ldots \ldots \ldots 35$

Marcus Marlene Marsh, $u$-mappings on trees $\ldots \ldots \ldots \ldots \ldots \ldots \ldots \ldots \ldots \ldots$

Peter Andrew Symonds, Localization in the classification of flat

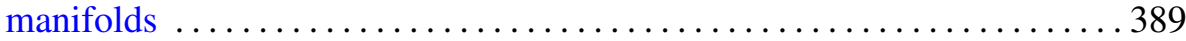

